\section{IAU Symposium}

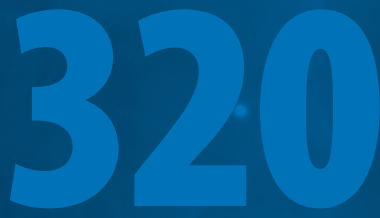

11-14 August 2015

Honolulu, United States

\section{Solar and Stellar Flares and their Effects on Planets}

Edited by

Alexander G. Kosovichev

Suzanne L. Hawley

Petr Heinzel

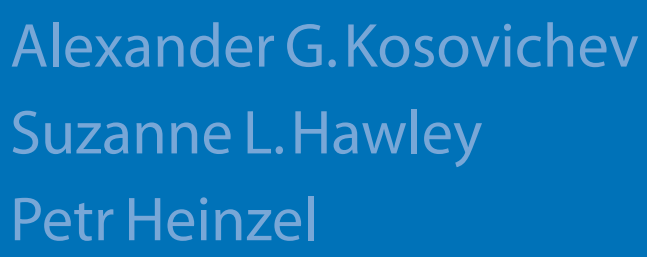

ISSN 1743-9213

International Astronomical Union

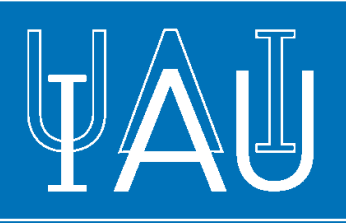


COVER ILLUSTRATION:

Image of a massive X1.9 class solar flare on the Sun on Nov. 3, 2011 at 20:27 UT, taken by the NASA's Solar Dynamics Observatory. The flare erupted from an extremely active region on the Sun called AR11339. 
IAU SYMPOSIUM PROCEEDINGS SERIES

\author{
Chief Editor \\ THIERRY MONTMERLE, IAU General Secretary \\ Institut d'Astrophysique de Paris, \\ 98bis, Bd Arago, 75014 Paris, France \\ montmerle@iap.fr
}

Editor

PIERO BENVENUTI, IAU Assistant General Secretary

University of Padua, Dept of Physics and Astronomy, Vicolo dell'Osservatorio, 3, 35122 Padova, Italy

piero.benvenuti@unipd.it 
INTERNATIONAL ASTRONOMICAL UNION

UNION ASTRONOMIQUE INTERNATIONALE

International Astronomical Union

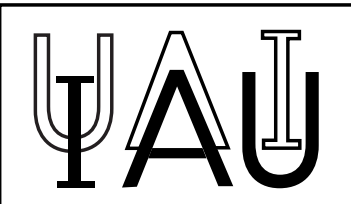

\title{
SOLAR AND STELLAR FLARES AND THEIR EFFECTS ON PLANETS
}

\author{
PROCEEDINGS OF THE 320th SYMPOSIUM \\ OF THE INTERNATIONAL ASTRONOMICAL \\ UNION HELD IN HONOLULU, UNITED STATES \\ AUGUST 11-14, 2015
}

Edited by

ALEXANDER G. KOSOVICHEV

New Jersey Institute of Technology, Newark, USA

SUZANNE L. HAWLEY

University of Washington, Seattle, USA

and

PETR HEINZEL

Astronomical Institute of the CAS, Ondřejov, Czech Republic 
CAMBRIDGE UNIVERSITY PRESS

University Printing House, Cambridge CB2 8BS, United Kingdom

32 Avenue of the Americas, New York, NY 10013, USA

10 Stamford Road, Oakleigh, Melbourne 3166, Australia

(C) International Astronomical Union 2016

This book is in copyright. Subject to statutory exception and to the provisions of relevant collective licensing agreements, no reproduction of any part may take place without the written permission of the International Astronomical Union.

First published 2016

Printed in the UK by Bell \& Bain, Glasgow, UK

Typeset in System $\mathrm{HT}_{\mathrm{EX}} 2_{\varepsilon}$

A catalogue record for this book is available from the British Library Library of Congress Cataloguing in Publication data

This journal issue has been printed on $\mathrm{FSC}^{\mathrm{TM}}$-certified paper and cover board. FSC is an independent, non-governmental, not-for-profit organization established to promote the responsible management of the world's forests. Please see www.fsc.org for information.

ISBN 9781107137578 hardback

ISSN 1743-9213 


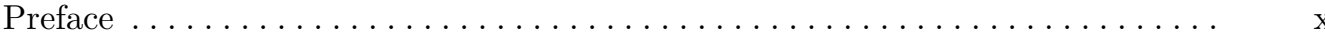

\section{Chapter 1: Introduction}

Solar and stellar flares and their impact on planets $\ldots \ldots \ldots \ldots \ldots \ldots \ldots$

K. Shibata

\section{Chapter 2: Multi-wavelength observations of solar flares}

Solar extreme ultraviolet (EUV) flare observations and findings from the Solar Dynamics Observatory (SDO) EUV Variability Experiment (EVE)....... T. N. Woods, F. G. Eparvier \& J. P. Mason

EUV irradiance observations from SDO/EVE as a diagnostic of solar flares .... R. O. Milligan

Fermi Large Area Telescope observation of high-energy solar flares: constraining

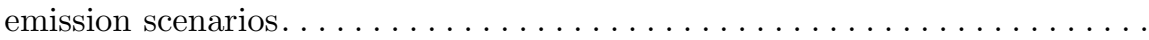

N. Omodei, M. Pesce-Rollins, V. Petrosian, W. Liu, F. R. da Costa \& A. Allafort, for the Fermi-LAT collaboration

Observations and modeling of the ultraviolet emission of solar flares . . . . . . . K. Mikuta, A. Berlicki \& P. Heinzel

The exceptional aspects of the confined X-class flares of solar active region 2192 J. K. Thalmann, Y. Su, M. Temmer \& A. M. Veronig

Spectroscopic UV observations of M 1.0 class solar flare from IRIS satellite. . . . V. M. Sadykov, A. G. Kosovichev, I. N. Sharykin \& S. V. Dominguez

GREGOR observations of a small flare above a sunspot $\ldots \ldots \ldots \ldots \ldots \ldots$ M. Sobotka, J. Dudik, C. Denker, H. Balthasar, J. Jurčák, W. Liu $\mathfrak{G}$ the GREGOR Team

On the fine structure of solar flare X-ray loop top sources............ T. Mrozek \& S. Kotomański

High-temperature solar flare plasma behaviour from crystal spectrometer

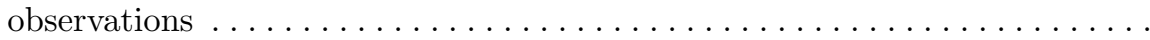

B. Sylwester, J. Sylwester, K. J. H. Phillips, A. Kepa \& T. Mrozek

Multitemperature analysis of solar flare observed on 2003 March 29 . . . . . . .

A. Kepa, B. Sylwester, J. Sylwester, M. Siarkowski, T. Mrozek \& M. Gryciuk

Model of flare lightcurve profile observed in soft X-rays. . . . . . . . . .

M. Gryciuk, M. Siarkowski, S. Gburek, P. Podgorski, J. Sylwester, Anna Kepa \& Tomasz Mrozek

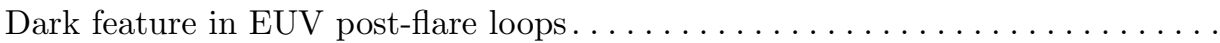
Q. Song, M. Zhang, J.-S. Wang, X.-S. Feng \& X.-X. Zhang

Restricted propagation of an "EIT wave" in the low solar corona.......... D. M. Long, D. Pérez-Suárez \& G. Valori 
Coronal quasi-periodic fast-propagating magnetosonic waves observed

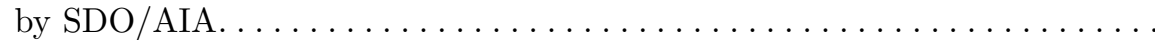

Y. Shen

Multi-wavelength observations of filament oscillations induced by shock waves . . $Y$. Shen

Solar flare soft X-ray spectra from Diogeness observations $\ldots \ldots \ldots \ldots \ldots \ldots$

M. Stȩślicki, J. Sylwester, B. Sylwester, Ż. Szaforz, Z. Kordylewski,

S. Ptocieniak, M. Siarkowski \& Kenneth J. H. Phillips

Thermal characteristics of a B8.3 flare observed on July $04,2009 \ldots \ldots \ldots \ldots \ldots$

A. K. Awasthi, B. Sylwester, J. Sylwester \& R. Jain

\section{Chapter 3: Advances in observations of stellar flares}

Discovery of superflares . . . . . . . . . . . . . . . . . . D. Nogami

The shape of $\mathrm{M}$ dwarf flares in Kepler light curves................. J. R. A. Davenport

The frequency of stellar X-ray flares from a large-scale XMM-Newton sample . . J. P. Pye ES S. R. Rosen

High dispersion spectroscopy of solar-type superflare stars with Subaru/HDS. . Y. Notsu, S. Honda, H. Maehara, S. Notsu, T. Shibayama, D. Nogami $\&$ K. Shibata

Statistical properties of superflares on solar-type stars based on the Kepler 1-min cadence data . . . . . . . . . . . . . . . . . . . . . . . . . .

H. Maehara, T. Shibayama, Y. Notsu, S. Notsu, S. Honda, D. Nogami \&s K. Shibata

Flares in A-type stars?

M. G. Pedersen, V. Antoci \& H. Korhonen

Flares from ultracool L dwarfs with Kepler.....................

J. E. Gizis, R. Paudel, P. K. G. Williams, A. J. Burgasser ES S. J. Schmidt

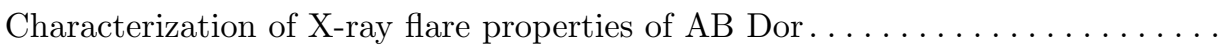
S. Lalitha

Ultraviolet spectrophotometry of flares on "quiescent" $\mathrm{M}$ and $\mathrm{K}$ dwarf exoplanet

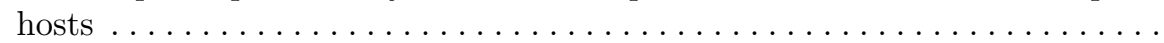
R. O. Parke Loyd, K. France \& A. Youngblood

\section{Chapter 4: Magnetic field structure and dynamics of flaring regions}

Nonlinear force-free modeling of magnetic fields in flare-productive active regions M. S. Wheatland \& S. A. Gilchrist

Hinode magnetic-field observations of solar flares for exploring the energy storage and trigger mechanisms $\ldots \ldots \ldots \ldots \ldots \ldots \ldots \ldots \ldots \ldots \ldots \ldots$

T. Shimizu, S. Inoue \& Y. Kawabata 
Slipping magnetic reconnection and complex evolution of a flux rope and flare

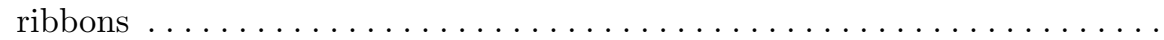

T. Li \& J. Zhang

Magnetic reconnection between an emerging active region and the quiet Sun ...

B. Zhang, J. Zhang, S. Yang, T. Li, Y. Zhang \& L. Li

Radio spectroscopy of stellar flares: magnetic reconnection \& CME shocks in stellar

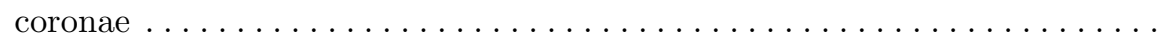

J. Villadsen, G. Hallinan \& S. Bourke

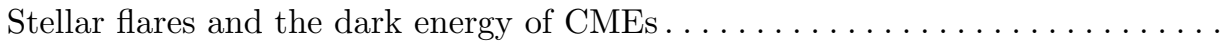
J. J. Drake, O. Cohen, C. Garraffo \& V. Kashyap

Evidence of thermal conduction suppression in hot coronal loops: supplementary

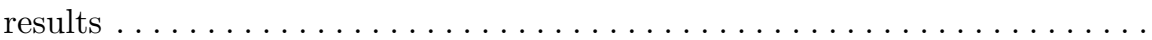

T. Wang, L. Ofman, X. Sun, E. Provornikova \& J. M. Davila

\section{Chapter 5: Flares and plasma eruptions}

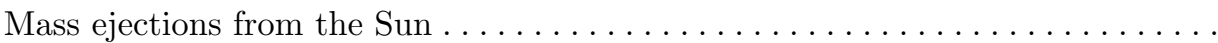

L. M. Green

Conditions for the existence of Kelvin-Helmholtz instability in a CME . . . . . . . A. Páez, V. Jatenco-Pereira, D. Falceta-Gonçalves \& M. Opher

Searching for failed eruptions interacting with overlying magnetic field . . . . . . .

D. Gronkiewicz, T. Mrozek, S. Kotomański \& M. Chruślińska

The estimate of hot Jupiter mass loss rate in the interaction with CME from a

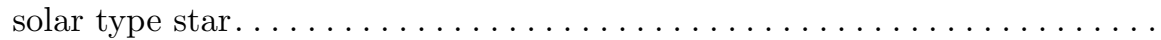

D. V. Bisikalo \& A. A. Cherenkov \& P. V. Kaygorodov

\section{Chapter 6: Particle acceleration and transport}

Numerical RHD simulations of flaring chromosphere with Flarix . . . . . . . . . . P. Heinzel, J. Kašparová, M. Varady, M. Karlický \& Z. Moravec

Response of chromospheric lines to different periodic non-thermal

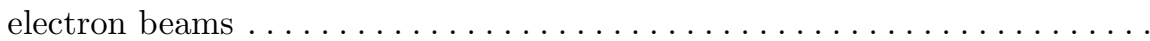
J. Cheng \& M. Ding

Updated calculations of the ionization equilibrium for the non-Maxwellian electron

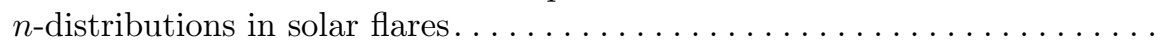

E. Dzif̌̌áková \&̛ J. Dudik

\section{Chapter 7: Comparison of solar and stellar flares}

Flare stars across the H-R diagram: a clue to the origin of the corona . . . . . . .

L. A. Balona

White-light continuum in stellar flares. . . . . . . . . . . . . .

A. F. Kowalski

White-light continuum emission from a solar flare and plage. . . . . . . .

A. Berlicki, A. K. Awasthi, P. Heinzel \& M. Sobotka 
Distinguishing between coronal cloud prominences and channel prominences and their associations with solar and stellar flares $\ldots \ldots \ldots \ldots \ldots \ldots \ldots$

S. F. Martin, O. Engvold, Y. Lin \& J. A. da Silva

On the origin of solar and stellar flares $\ldots \ldots \ldots \ldots \ldots \ldots \ldots \ldots \ldots \ldots \ldots \ldots \ldots \ldots$

S. Ibadov \& F. S. Ibodov

\section{Chapter 8: Solar and stellar magnetic activity}

The role of complex magnetic topologies on stellar spin-down. . . . . . . .

V. Réville, A. S. Brun, A. Strugarek, S. P. Matt, J. Bouvier, C. P. Folsom \& P. Petit

Synthetic activity indicators for M-type dwarf stars. . . . . . . . . .

S. Wedemeyer \& H.-G. Ludwig

Super-active regions in solar cycle $24 \ldots \ldots \ldots \ldots \ldots \ldots \ldots \ldots \ldots \ldots \ldots \ldots \ldots \ldots \ldots \ldots \ldots$

A. Chen \& J. Wang

Solar activities observed with the New Vacuum Solar Telescope............ S. Yang \& J. Zhang

Lightcurve studies and magnetic activities of several eclipsing binaries . . . . . .

X. L. Han, L. Zhang, Q. Pi \& D. Wang

The design of solar synoptic chart for space weather forecast $\ldots \ldots \ldots \ldots \ldots$ Q. Song, J.-S. Wang, X.-S. Feng \& X.-X. Zhang

Descriptive study of X-class flares released in the year 2014, during the double peak

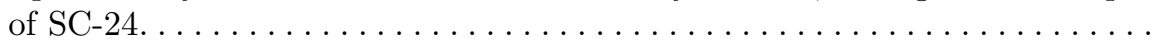

A. A. Hady, M. H. Mostafa \& S. W. Samwel

Temporal solar irradiance variability analysis using neural networks . . . . . . . A. Tebabal, B. Damtie \& M. Nigussie

The Reflecting Heliometer of Rio de Janeiro after 6 years of activity . . . . . . . .

S. C. Boscardin, C. Sigismondi, J. L. Penna, V. D'Avila, E. Reis-Neto \& A. H. Andrei

Solar radius variations: new look on the wavelength dependence. . . . . . . . J.-P. Rozelot, A. Kosovichev \& A. Kilcik

Solar diameter measurements from eclipses as a solar variability proxy . . . . . . D. W. Dunham, S. Sofia, K. Guhl \& D. Herald

\section{Chapter 9: Flares and star-planet interaction}

Magnetism and activity of planet hosting stars. . . . . . . . . . .

J. T. Wright \& B. P. Miller

Stellar wind - magnetosphere interactions in hot Jupiters

D. L. Buzasi

Ultraviolet and X-ray irradiance and flares from low-mass exoplanet host stars .

K. France, R. O. P. Loyd \& A. Brown 
Optical hydrogen absorption consistent with a bow shock around the hot Jupiter

HD 189733 b. . . . . . . . . . . . . . . . . . . . . . . . . . . . . . .

P. W. Cauley, S. Redfield, A. G. Jensen, T. Barman, M. Endl \&

W. D. Cochran

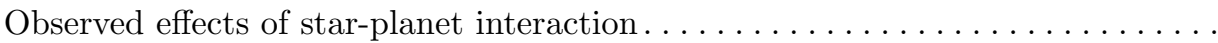

S. J. Wolk \& I. Pillitteri \& K. Poppenhaeger

CARMENES: M dwarfs and their planets . . . . . . . . . . . . . . .

A. Quirrenbach, P. J. Amado, J. A. Caballero, H. Mandel, R. Mundt,

A. Reiners, I. Ribas, W. Seifert, M. Azzaro, D. Galadi \& the CARMENES Consortium

Quiescent and flaring Lyman- $\alpha$ radiation of host stars and effects on exoplanets J. L. Linsky, K. France, Y. Miguel \& L. Kaltenegger

The particle and magnetic environments surrounding close-in exoplanets . . . . . A. A. Vidotto, R. Fares, M. Jardine, C. Moutou \& J.-F. Donati

Magnetic energy fluxes in close-in star-planet systems . . . . . . . . . . . A. Strugarek, A. S. Brun, S. P. Matt \& V. Réville

The early Earth under a superflare and super-CME attack: prospects for life .. . V. Airapetian, A. Glocer \& G. Gronoff

\section{Chapter 10: New frontiers in solar and stellar flares and research programs}

Synergy between solar and stellar flares: challenges and perspectives . . . . . . . S. L. Hawley

First radio burst imaging observation from Mingantu Ultrawide Spectral Radiohe-

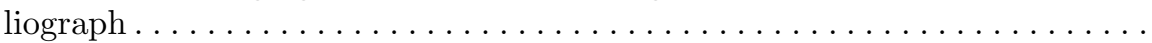

Y. Yan, L. Chen, S. Yu \& CSRH Team

The Lyman- $\alpha$ Solar Telescope (LST) for the ASO-S mission . . . . . . . . . . H. $\mathrm{Li}$

Geant4 simulations of STIX Caliste-SO detector's response to solar X-ray radiation J. Barylak, A. Barylak, T. Mrozek, M. Steślicki, P. Podgórski \& H. Netzel

Solar X-rays from 0.3 A.U.: The ChemiX Bragg Spectrometer on Interhelioprobe J. Sylwester, M. Siarkowski, J. Bakata, Z. Szaforz, M. Kowaliński, M. Steślicki, B. Sylwester, Z. Kordylewski, O. Dudnik, V. D. Kuznetsov, V. Polansky, S. Kuzin \& K. J. H. Phillips

Progress of site survey for large solar telescopes in western China . . . . . . . .

Y. Liu, T. Song, X. Zhang, S. Liu, M. Zhao, Z. Tian, Y. Miao, H. Li, J. Huang, B. Su, Y. Lu, X. Li \& Q. Song

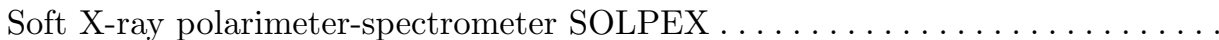

M. Stȩślicki, J. Sylwester, S. Ptocieniak, J. Bakata, Ż. Szaforz,

D. Ścistowski, M. Kowaliński, J. Hernandez, S. Kuzin \& S. Shestov

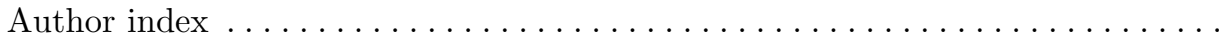




\section{Preface}

Recent advances in observations and modelling of solar and stellar flares have opened new perspectives for understanding of the fundamental physical mechanisms of magnetic energy storage and release, particle acceleration, and radiative and dynamical processes in solar and stellar flares. The new interest in this topic is stimulated by Kepler observations which have led to the discovery that stellar flares occur not only in M-type dwarfs (UV Ceti-type variables) but also in a wide range of A-F type stars. Previously, it was believed that the F- and A-type stars do not have flaring activity. The discovery of super-flares on solar-type stars has raised questions about the possibility of such flares on the Sun, and led to hot debates about the potential effects of such superflares on terrestrial and extra-terrestrial planets, including their impact on the origin and evolution of life. These results triggered new interest in the physical mechanism of solar and stellar flares, and their connection with the dynamo mechanism and stellar properties.

Recent observations of solar flares from the Solar Dynamics Observatory (SDO), IRIS, RHESSI, STEREO, Hinode and Fermi space observatories and large ground-based telescopes have revealed details of the magnetic topology of flaring active regions, obtained important insight into the processes of magnetic reconnection and particle acceleration, and led to new understanding of the importance of local response and global-scale coupling of the flare dynamics. Among the new observations, the Fermi detection of extremely long gamma-ray emission in many solar flares is particularly surprising. These and other new results challenge the current theories of solar and stellar flares, and even cause to reconsider the whole, once well-established, paradigm about the common physical origin and properties of the solar and stellar flares.

Previously, stellar flares associated with active M-type stars were thought to be similar to solar flares representing a sudden release of magnetic energy accumulated in the coronal part of sunspot region, in the form of high-energy particles which heat the atmosphere and corona. Stellar flares can be four orders of magnitude more powerful, which is thought be due to bigger starspot regions generated by a more efficient dynamo process, because many of the flaring stars rotate faster than the Sun. However, the new observations have raised an alternative point of view that such powerful flares may be due to the interaction with close companions - 'hot Jupiters'. The discovery of flares on hot A-type stars with a very shallow outer convection zone and without strong magnetic field causes additional problems with the dynamo origin of the flare energy on these stars.

The IAU Symposium 320 had been a forum for discussing the recent advances in observations and theories of solar and stellar flares, focused on the understanding of their phenomenological and physical aspects, as well as consequences for terrestrial planets and exoplanets. It covered a broad range of phenomena, from the magnetic topology of flares and mechanisms of impulsive energy release to high-energy flare emission and potential impacts on planets.

The papers presented in the Proceedings are focused on recent advances in multiwavelength observations with new space and ground-based telescopes, as well as in theory and numerical simulations. This includes discussions of similarity and differences in magnetism of the Sun and flaring stars, links to the interior dynamics and surface magnetism, physical mechanisms of magnetic energy storage and release, particle acceleration, properties of impulsive radio, optical, EUV, X-ray and gamma-ray emissions, shocks and mass ejections, origin of superflares on solar-types stars, and impact of flares 
on planetary atmospheres. We hope that this volume will be useful to senior and new researchers in this fascinating and rapidly developing field of astronomy.

Alexander G. Kosovichev, Suzanne Hawley, and Petr Heinzel, co-chairs SOC 